\title{
Dynamical Analysis and Microcontroller Implementation of Linear Resistor-Capacitor Shunted Josephson Junction Model
}

\author{
Dianorré Tokoue Ngatcha ${ }^{(\mathbb{D} *, 1}$, Alhadji Abba Oumate ${ }^{\mathbb{D}} \beta, 2$, Alex Stephane Kemnang Tsafack ${ }^{(D)} \gamma$ and Sifeu Takougang Kingni ${ }^{(\mathbb{D})} \S, 4$ \\ * Department of Automotive and Mechatronics Engineering, National Higher Polytechnic School of Douala, University of Douala, Box 24, 2701 Douala, \\ Cameroon, ${ }^{\beta}$ Department of Physics, Faculty of Science, University of Maroua, P.O. Box 814, Maroua, Cameroon, ${ }^{\gamma}$ Research unit of Condensed Matter of \\ Electronics and Signal Processing, Department of Physics, Faculty of Sciences, University of Dschang, P. O. Box 67, Dschang, Cameroon, \$Department of \\ Mechanical, Petroleum and Gas Engineering, Faculty of Mines and Petroleum Industries, University of Maroua, P.O. Box 46, Maroua, Cameroon.
}

ABSTRACT Theoretical analysis and microcontroller implementation of linear resistor-capacitor shunted Josephson junction (LRCSJJ) model are studied in this paper. The rate-equations describing the LRCSJJ model has no or two equilibrium points. One of the equilibrium points is a saddle node and the other one is a stable node. The hysteresis loop of current-voltage curves increases with the rising of the capacitance of Josephson junction (JJ). Excitable mode, limit cycle, periodic and chaotic behaviors are found in LRCSJJ model with external alternative current $(A C)$ source thanks to the two modulation parameters largest Lyapunov exponents (LLE) diagram. LRCSJJ model exhibits two different shapes of chaotic attractors by varying the modulation amplitude. Finally, the existence of chaotic behaviors is confirmed by microcontroller results obtained from the microcontroller implementation of LRCSJJ model.

\section{KEYWORDS}

Chaos

Josephson junction

Hysteresis loop

Excitable mode

Microcontroller

implementation.

\section{INTRODUCTION}

Josephson junction devices have been studied by many researchers because they are very good candidates commonly exploited for the construction of complex systems for specific applications (Levi et al. 1978; Kautz and Monaco 1985; McCumber 1968; Zhang et al. 2011; Malishevskii and Uryupin 2020). In the literature, there is two main electrical rate-equations of JJ: Resistor and capacitor shunted JJ (RCSJJ) mode (Cawthorne et al. 1998) and resistor, capacitor and inductive shunted JJ (RCISJJ) model (Dana et al. 2006; Whan et al. 1995). Typically by using RCSJJ model to describe JJ, resulted in a fairly good agreement with experiment. Nonetheless, it was not able to generate significant features of experimental currentvoltage characteristics. Better agreement with experiment is found when the RCSJJ model is adopted by inserting an inductor in series with the shunt resistor in order to obtain the RCLSJJ model

\footnotetext{
Manuscript received: 8 June 2021,

Revised: 14 July 2021,

Accepted: 21 July 2021.

1 tokouengatcha1@gmail.com

2 oumatealhadji.ao@gmail.com

${ }^{3}$ alexstephanekemnang@gmail.com (Corresponding Author)

${ }^{4}$ stkingni@gmail.com
}

(Cawthorne et al. 1998; Dana et al. 2006; Whan et al. 1995; Stewart 1968; Neumann and Pikovsky 2003; Takougang Kingni et al. 2017).

In fact, there is four models of JJ namely: Nonlinear RCSJJ model(Levi et al. 1978; Likharev 1986), LRCSJJ model (Salam and Sastry 1985; Bartuccelli et al. 1986),nonlinear RCLSJJ model (Cawthorne et al. 1998; Dana et al. 2006; Whan et al. 1995; Stewart 1968), and linear RCISJJ model (Neumann and Pikovsky 2003; Takougang Kingni et al. 2017) have been reviewed to check if a JJ device can be used as a transmitter and receiver in chaos based communications. The two RCSJJ models show chaotic behaviors when driven by external sinusoidal current source (Levi et al. 1978; Likharev 1986; Salam and Sastry 1985; Bartuccelli et al. 1986) whereas the two RCISJJ models generated chaotic behaviors with external DC (Cawthorne et al. 1998; Dana et al. 2006; Whan et al. 1995; Stewart 1968; Neumann and Pikovsky 2003; Takougang Kingni et al. 2017; Dana et al. 2001).The RCISJ models have been revealed more appropriate in high-frequency applications (Cawthorne et al. 1998; Dana et al. 2006; Whan et al. 1995; Stewart 1968; Neumann and Pikovsky 2003; Takougang Kingni et al. 2017). In (Dana et al. 2006), Dana et al. have been studied how the chaos found in nonlinear RCLSJJ model could be applied as a chaos generator for communications.

The authors of (Takougang Kingni et al. 2017) have been studied 
the dynamical analysis of linear RCISJJ model and its fractionalorder form as well as its application to digital cryptography. Synchronization of two coupled linear RCISJJ models via cyclic coupling and its microcontroller-based implementation was reported by Ojo and al (Ojo et al. 2019). Few research works have been reported on RCSJJ models (Levi et al. 1978; Likharev 1986; Salam and Sastry 1985; Bartuccelli et al. 1986; Kautz and Monaco 1985). Salam and Shastry have been studied the dynamical behaviors of LRCSJJ model actuated both by direct current (DC) and AC with emphasis on the AC case (Salam and Sastry 1985). In (Bartuccelli et al. 1986), Chaos in LRCSJJ model driven AC have been investigated by using the Melnikov method. Chaotic behavior in LRCSJJ model driven by an AC have been studied through digital simulations by Kantz and Monaco (Kautz and Monaco 1985). McCumber have been investigated on the alternative current impedance and he saw the influence both in the response time and the DC voltage-current characteristics (McCumber 1968).

This paper studies the dynamical behavior and the microcontroller implementation of the LRCSJJ model. The paper is organized as follows: Theoretical analysis of LRCSJJ model is studied in Section 2. Microcontroller implementation of the LRCSJJ model is investigated in Section 3. Section 4 presents the conclusion.

\section{DYNAMICAL ANALYSIS OF LRCSJJ MODEL}

LRCSJJ model (Kautz and Monaco 1985) is represented in Fig. 1.

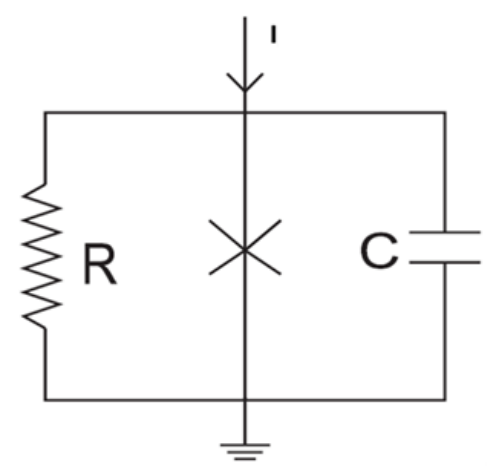

Figure 1 Schematic view of LRCSJJ model.

The circuit of Fig. 1 consists of the external current source $I$, capacitor $C$, linear resistor $R$ and JJ element connected in parallel. A voltage $V$ is developed across the $\mathrm{JJ}$ due to the application of by applying the Kirchhoff law, the following equations are obtained:

$$
\begin{gathered}
v=\frac{h}{2 \pi e} \frac{d \phi}{d t^{\prime}} \\
C \frac{d \mathrm{v}}{d t^{\prime}}+\frac{\mathrm{v}}{R}+I_{J}=I
\end{gathered}
$$

where $v$ is the voltage, $h$ is the Planck constant, $t^{\prime}$ is the time, $e$ is the electron charge, $I_{J}=I_{J C} \sin \phi$ is the JJ current and $\phi=\phi_{2}-\phi_{1}$ is the phase difference. By introducing the following parameters $t=\omega_{0} t^{\prime}, i(t)=I / I_{C J}, \omega_{0}=\left(2 \pi e I_{J C} / h C\right)^{1 / 2}$, $V=\mathrm{v} \sqrt{2 \pi e C / h I_{C J}}$ and $\beta_{C}=2 \pi e R^{2} C I_{C J} / h>0$, the set of Eqs. $(1,2)$ can be normalized as:

$$
\begin{gathered}
\frac{d \phi}{d t}=\mathrm{V} \\
\frac{d V}{d t}=i(t)-\mathrm{V} / \sqrt{\beta_{C}}-\sin (\phi)
\end{gathered}
$$

where $\beta_{C}$ is the capacitance of JJ

Analytical and numerical analysis of LRCSJJ model driven by external DC source

The external current source is considered as a DC: $i(t)=i_{d c}$, LRCSJJ model displays two equilibrium points $E_{1}=\left(\arcsin \left(i_{d c}\right), 0\right)$ and $E_{2}=\left(\pi-\arcsin \left(i_{d c}\right), 0\right)$ for $i_{d c} \leq 1$ whereas it has noequilibrium point for $i_{d c}>1$. The characteristic equation associated to the equilibrium point $E_{1}=\left(\arcsin \left(i_{d c}\right), 0\right)$ is

$$
\lambda^{2}+\frac{1}{\sqrt{\beta_{C}}} \lambda+\sqrt{1-i^{2}}=0
$$

and the eigenvalues are

$$
\lambda_{1}=\frac{1}{2 \sqrt{\beta_{C}}}\left(-1+\sqrt{1-4 \beta_{C} \sqrt{1-i_{d c}^{2}}}\right)
$$

and

$$
\lambda_{2}=\frac{1}{2 \sqrt{\beta_{C}}}\left(-1-\sqrt{1-4 \beta_{C} \sqrt{1-i_{d c}^{2}}}\right) .
$$

Since $\lambda_{1}<0$ and $\lambda_{2}<0$, the equilibrium point $E_{1}=$ $\left(\arcsin \left(i_{d c}\right), 0\right)$ is a stable node. The characteristic equation at the equilibrium point $E_{2}=\left(\pi-\arcsin \left(i_{d c}\right), 0\right)$ is

$$
\lambda^{2}+\frac{1}{\sqrt{\beta_{C}}} \lambda-\sqrt{1-i_{d c}^{2}}=0
$$

and the eigenvalues are

$$
\lambda_{1}=\frac{1}{2 \sqrt{\beta_{C}}}\left(-1+\sqrt{1+4 \beta_{C} \sqrt{1-i_{d c}^{2}}}\right)
$$

and

$$
\lambda_{2}=\frac{1}{2 \sqrt{\beta_{C}}}\left(-1-\sqrt{1+4 \beta_{C} \sqrt{1-i_{d c}^{2}}}\right) .
$$

Since $\lambda_{1}>0$ and $\lambda_{2}<0$, the equilibrium point $E_{2}=(\pi-$ $\left.\arcsin \left(i_{d c}\right), 0\right)$ is a saddle node.

Figure 2 presents the current-voltage curve for given values of the capacitance of $\mathrm{JJ}$.

When the DC $i(t)=i_{d c}$ increases (black line in Fig. 2), the trajectories of voltage exhibits excitable mode for $i(t)=i_{d c} \leq 1.0$. The voltage exhibits period-1-oscillations for $i(t)=i_{d c}>1.0$. By decreasing DC $i(t)=i_{d c}$ (see red line in Fig. 2), the voltage depicts the similar dynamical behaviors as in Fig. 2 (black line). By comparing the two sets of data [for increasing (black line) and decreasing (red line)] used to plot Fig. 2, there is a region of the DC called hysteresis loop where the voltage $V$ exhibis the similar dynamical behaviors as seen in Fig. 2 (black line) but the amplitudes of the voltage $V$ are differents. So, LRCSJJ model shows bistability phenomenon in the hysteresis loop. By increasing the capacitance of $\mathrm{JJ} \beta_{\mathrm{C}}$, the hysteresis loop increases.

\section{Numerical analysis of LRCSJJ model driven by external AC source}

In this subsection, we consider the external current source is to be an AC: $i(t)=i_{d c}+i_{m} \sin \left(\omega_{m} t\right)$ where the parameters $i_{d c}, i_{m}$ and $\omega_{m}$ are DC, modulation current and modulation pulsation, respectively. The two modulation parameters LLE diagram of the LRCSJJ model is illustrated in Fig. 3. 

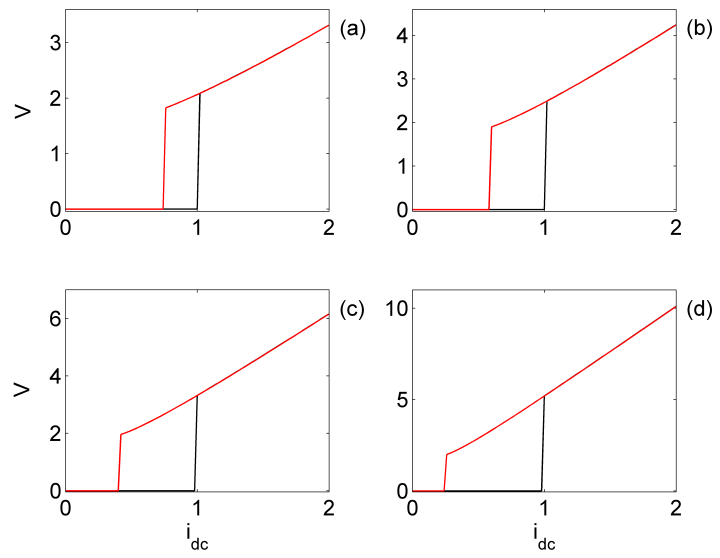

Figure 2 Current-voltage curve for given values of $\beta_{C}$ : (a) $\beta_{C}=$ 2.25 , (b) $\beta_{C}=4$, (c) $\beta_{C}=9$ and (d) $\beta_{C}=25$. The current-voltage curves are found by increasing (black line) and decreasing (red line) the DC $i=i_{d c}$.

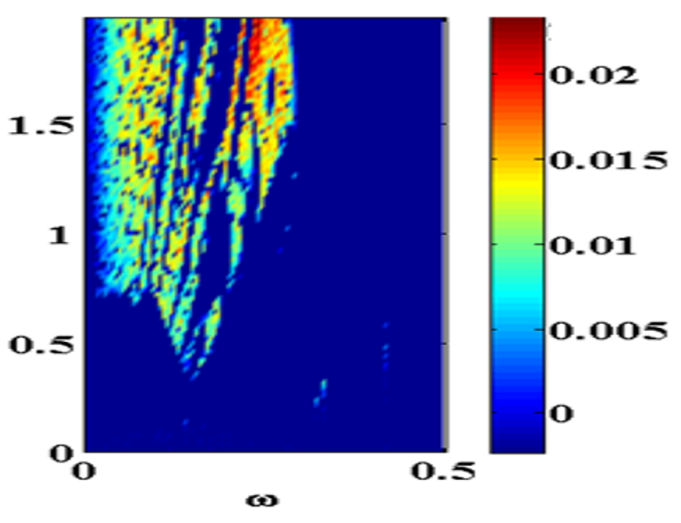

Figure 3 Two parameters LLE diagram of the LRCSJJ model in $\left(\omega, i_{m}\right)$ space for $i_{d c}=0.3$ and $\beta_{C}=25$.
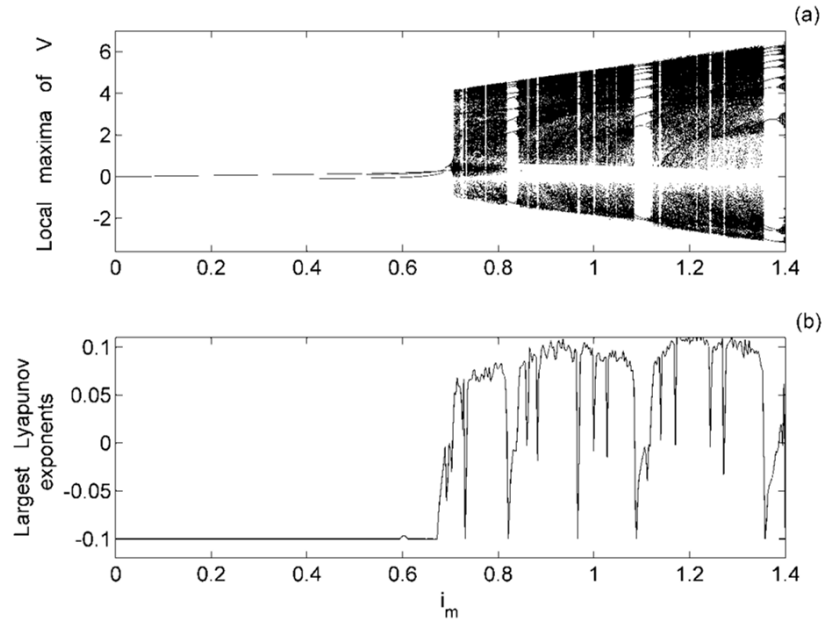

Figure 4 Bifurcation diagrams of $V(t)$ (a) and LLE (b) versus the parameter $i_{m}$ for $i_{d c}=0.3, \omega_{m}=0.25$ and $\beta_{C}=25$.
Excitable mode, periodic behaviors and chaotic behaviors regions are illustrated in Fig. 3. The bifurcation diagram versus the parameter $i_{m}$ and LLE are depicted in Fig. 4 for $\omega_{m}=0.25$.

By increasing the amplitude of modulation $i_{m}$ from 0.0001 to 1.4, the voltage $V(t)$ in Fig. 4(a) displays limit cycle, period-2oscillation, period-3-oscillation, period-2-oscillations then perioddoubling bifurcation to chaos encrusted with periodic windows. The LLE of Fig. 4(b) confirms the results of Fig. 4(a). Figure 5 depicts the chaos obtained in Fig. 4 for given values of parameter $i_{m}$.
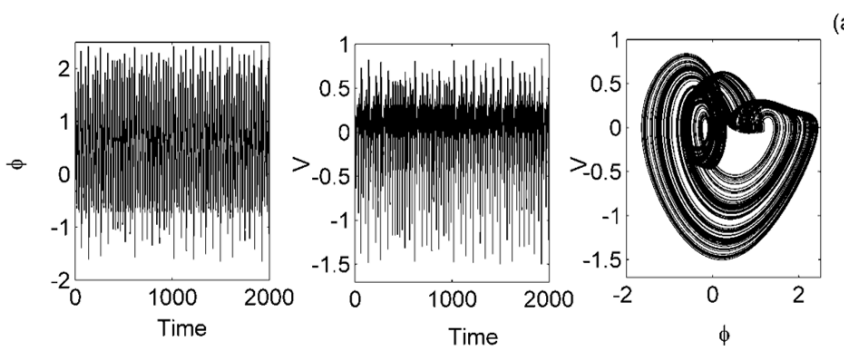

(a)
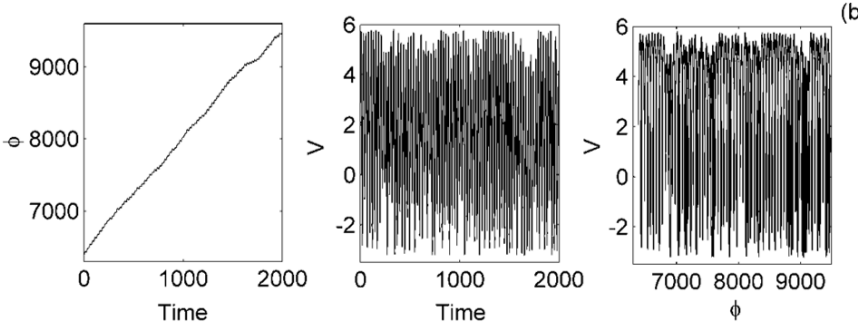

Figure 5 Time histories of $\phi, V$, and phase planes for given values of parameter $i_{m}: i_{m}=0.705$ and $i_{m}=1.2$, are $i_{d c}=0.3, \omega_{m}=$ 0.25 and $\beta_{C}=25$. The initial conditions are $(\phi(0)=0, V(0)=0)$.

For $i_{m}=0.705$, the phase difference and the voltage exhibit chaotic attractors as seen in Fig. 5(a). While in Fig. 5(b) for $i_{m}=1.2$, the phase difference does not depict chaotic oscillations and the voltage displays chaotic oscillations. The phase portraits in Fig. 5 shows two different shapes of chaotic attractors for two given values of amplitude of modulation $i_{m}$.

\section{MICROCONTROLLER IMPLEMENTATION OF LRCSJJ MODEL}

The microcontroller implementation of LRCSJJ model is presented in Fig. 6.

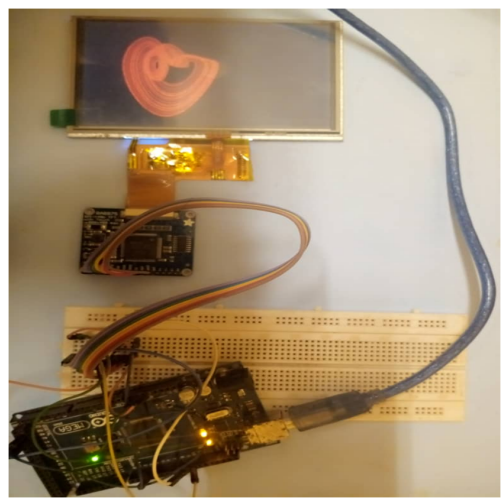

Figure 6 Microcontroller design circuit of the LRCSJJ model. 
The microcontroller implementation of RCSJJ model shown in Fig. 6 is based on the Arduino. Fig. 7 presents the time series and phase portraits obtained from the microcontroller implementation of the LRCSJJ model.

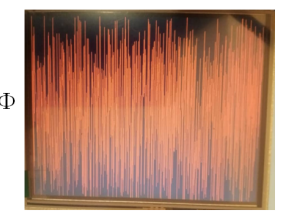

Time

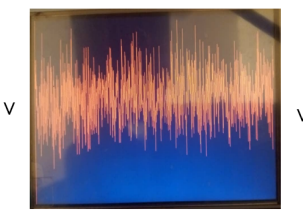

Time

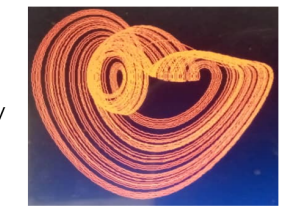

$\Phi$
Figure 7 Time series and phase portrait obtained from the microcontroller implementation of LRCSJJ model.

The microcontroller results of Fig. 7 are qualitatively matched with the results of Fig. 5(a). Thus, the existence of chaotic behaviors found in LRCSJJ model is confirmed.

\section{CONCLUSION}

This paper deals with the theoretical analysis and microcontroller implementation of linear resistor-capacitor shunted Josephson junction model. The rate-equations describing the linear resistivecapacitive shunted Josephson junction model has two or no equilibrium points relying on the external direct current source. The stability analysis of the two equilibrium points of linear resistorcapacitor shunted Josephson junction model was investigated. The increasing of the capacitance of Josephson junction leaded to an increased in the hysteresis loop of current-voltage curves. For given modulation parameters of external current source, linear resistorcapacitor shunted Josephson junction model displayed two different shapes of chaotic attractors, periodic attractors, limit cycle and excitable mode. The existence of chaotic behaviors was confirmed by microcontroller results obtained from the microcontroller implementation of linear resistor-capacitor shunted Josephson junction model.

\section{Conflicts of interest}

The authors declare that there is no conflict of interest regarding the publication of this paper.

\section{LITERATURE CITED}

Bartuccelli, M., P. L. Christiansen, N. F. Pedersen, and M. P. Sørensen, 1986 Prediction of chaos in a josephson junction by the melnikov-function technique. Physical Review B 33: 4686.

Cawthorne, A., C. Whan, and C. Lobb, 1998 Complex dynamics of resistively and inductively shunted josephson junctions. Journal of applied physics 84: 1126-1132.

Dana, S. K., D. C. Sengupta, and K. D. Edoh, 2001 Chaotic dynamics in josephson junction. IEEE Transactions on Circuits and Systems I: Fundamental Theory and Applications 48: 990-996.

Dana, S. K., D. C. Sengupta, and C.-K. Hu, 2006 Spiking and bursting in josephson junction. IEEE Transactions on Circuits and Systems II: Express Briefs 53: 1031-1034.

Kautz, R. L. and R. Monaco, 1985 Survey of chaos in the rf-biased josephson junction. Journal of applied physics 57: 875-889.

Levi, M., F. C. Hoppensteadt, and W. Miranker, 1978 Dynamics of the josephson junction. Quarterly of Applied Mathematics 36: 167-198.

Likharev, K., 1986 Dynamics of josephson junctions and circuits gordon and breach. New York p. 92.
Malishevskii, A. and S. Uryupin, 2020 Current-voltage characteristics of radiating josephson sandwich. Physics Letters A 384: 126515.

McCumber, D., 1968 Effect of ac impedance on dc voltage-current characteristics of superconductor weak-link junctions. Journal of Applied Physics 39: 3113-3118.

Neumann, E. and A. Pikovsky, 2003 Slow-fast dynamics in josephson junctions. The European Physical Journal B-Condensed Matter and Complex Systems 34: 293-303.

Ojo, K., A. Adelakun, and A. Oluyinka, 2019 Synchronisation of cyclic coupled josephson junctions and its microcontroller-based implementation. Pramana 92: 1-7.

Salam, F. and S. Sastry, 1985 Dynamics of the forced josephson junction circuit: the regions of chaos. IEEE transactions on circuits and systems 32: 784-796.

Stewart, W., 1968 Current-voltage characteristics of josephson junctions. Applied physics letters 12: 277-280.

Takougang Kingni, S., G. Fautso Kuiate, R. Kengne, R. Tchitnga, and P. Woafo, 2017 Analysis of a no equilibrium linear resistive-capacitive-inductance shunted junction model, dynamics, synchronization, and application to digital cryptography in its fractional-order form. Complexity 2017.

Whan, C., C. Lobb, and M. Forrester, 1995 Effect of inductance in externally shunted josephson tunnel junctions. Journal of applied physics 77: 382-389.

Zhang, Z., K. Chau, Z. Wang, F. Li, and J. Li, 2011 Analysis of chaos in josephson junctions with external magnetic field for high-precision voltage measurement in electric vehicles. IEEE transactions on applied superconductivity 22: 4904704-4904704.

How to cite this article: Ngatcha, D. T., Oumate, A. A., Kemnang Tsafack A. S., and Kingni, S. T. Dynamical analysis and microcontroller implementation of linear resistor-capacitor shunted Josephson junction model. Chaos Theory and Applications, 3(2), 55-58, 2021. 